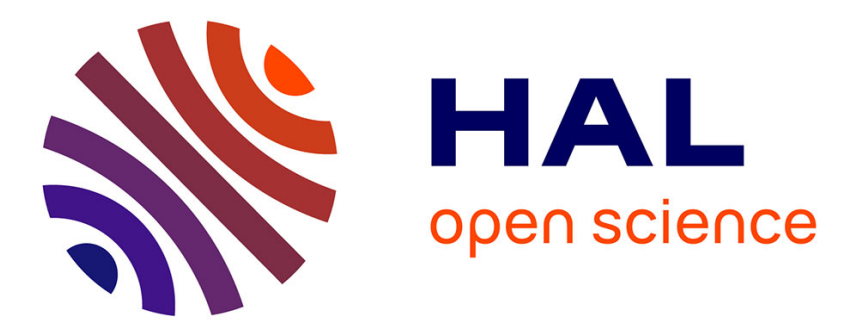

\title{
Les malaises adressés aux urgences sont fréquents, bénins et coûteux : étude épidémiologique des facteurs de risque d'hospitalisation en vue de désencombrer les urgences
}

E Gedda, A Robbins, M Hentzien, A Giltat, V Pinel-Petit, J Souille, Y N'guyen, Y N'Guyen

\section{To cite this version:}

E Gedda, A Robbins, M Hentzien, A Giltat, V Pinel-Petit, et al.. Les malaises adressés aux urgences sont fréquents, bénins et coûteux: étude épidémiologique des facteurs de risque d'hospitalisation en vue de désencombrer les urgences. La Revue de Médecine Interne, 2017, 38, pp.8 - 16. 10.1016/j.revmed.2016.08.013 . hal-03267096

\section{HAL Id: hal-03267096 \\ https://hal.science/hal-03267096}

Submitted on 29 Jun 2021

HAL is a multi-disciplinary open access archive for the deposit and dissemination of scientific research documents, whether they are published or not. The documents may come from teaching and research institutions in France or abroad, or from public or private research centers.
L'archive ouverte pluridisciplinaire HAL, est destinée au dépôt et à la diffusion de documents scientifiques de niveau recherche, publiés ou non, émanant des établissements d'enseignement et de recherche français ou étrangers, des laboratoires publics ou privés. 


\title{
Article Original REVMED-D-15-00342 R1
}

La revue de médecine interne

Les malaises adressés aux urgences sont fréquents, bénins et coûteux : étude épidémiologique des facteurs de risque d'hospitalisation en vue de désencombrer les urgences. (4182 mots)

Faintness in Emergency Departments are frequent, benign but expensive: an epidemiologic study of hospitalization's risk factors to reduce overcrowdings of Emergency Departments.

\author{
Authors : E. Gedda, ${ }^{a, b}$ A. Robbins, ${ }^{b}$ M. Hentzien, ${ }^{b}$ A. Giltat, ${ }^{b}$ \\ V. Pinel-Petit, ${ }^{a}$ J. Souille, ${ }^{a}$ Y. N'Guyen ${ }^{b^{*}}$
}

${ }^{\text {a }}$ Service d'accueil des urgences, Hôpital Maison Blanche, 51 rue Cognacq Jay, 51100 Reims

${ }^{\mathrm{b}}$ Service de médecine interne, maladies infectieuses et immunologie clinique, Hôpital Robert Debré, Avenue du Général Koenig, 51100 Reims

* auteur correspondant: Service de Médecine Interne, Maladies Infectieuses et Immunologie Clinique, Hôpital Robert Debré, Avenue Général Koenig, 51100 Reims. Tel +33 3267894 22. Fax +33 3267840 90. Mail: yohan.nguyen@wanadoo.fr.

Mots clés: Syncope; Perte de conscience; Service des Urgences médicales.

Keywords: Syncope; faintness; Loss of Consciousness; Emergency Department. 


\section{Résumé (248 mots) :}

Introduction: Nous avons tenté d'apprécier (i) la fréquence des consultations pour malaise au service d'accueil des urgences (SAU) d'un centre hospitalier universitaire (CHU), (ii) l'épidémiologie clinique et (iii) le coût des malaises, en s'intéressant aux facteurs associés à l'hospitalisation.

Méthodes : Cette étude rétrospective a été menée à partir des données des patients ayant consulté pour «malaise » au SAU du CHU de Reims, (01/01/12-31/03/12). Tous les dossiers ont été reclassés en syncope/lipothymie/perte de connaissance brève d'une part et en syncope selon la définition anglo-saxonne reprise par la Haute Autorité de Santé (HAS) d'autre part.

Résultats : Trois cent quarante et un patients parmi les 5953 patients de la période de l'étude (5,7\%) ont consulté pour malaise. Les données de 296 patients ont été analysées. Cette population comportait $62,8 \%$ de femmes, avec un âge médian de 43 ans. L'examen clinique était normal dans $57 \%$ des cas. Il s'agissait de lipothymies sans perte de connaissance complète, non prises en compte dans la classification HAS dans $48 \%$ des cas. Les patients restaient une durée médiane de 4 heures au SAU et 67 patients $(22,6 \%)$ ont été hospitalisés. Le coût minimal a été estimé à 280000 euros. Les facteurs de risque indépendamment associés à une hospitalisation étaient un âge $\geq 60$ ans ou la présence d'une perte de connaissance complète, à l'inverse de la présence de circonstances favorisant l'hypertonie vagale.

Conclusion : Un âge $\geq 60$ ans et/ou la présence d'une perte de connaissance complète semblent associés à l'hospitalisation. 


\section{Abstract (217 words):}

Introduction: We assessed (i) the frequency of consultations for faintness in the Emergency department (ED) of a university hospital centre (UHC), (ii) clinical epidemiology and (iii) cost of faintness, taking a particular interest into the determining risk factors for hospitalization.

Methods: This epidemiological study has been conducted retrospectively, from data obtained for every patient having consulted for faintness in ED of Reims UHC (01/01/12-03/31/12). Every medical record was classified as syncope/lipothymia/brief consciousness loss on one hand and as syncope according to the definition of the French Health High Authority (FHHA).

Results: Three hundred and forty one patients out of $5953(5,7 \%)$ were referred for faintness during the study period. Medical records were analysed for 296 patients. Sixty-two point eight percent were women, with a median age of 43 years. Physical examination was normal for $57 \%$ of patients. For $48 \%$ of cases, there was no complete consciousness loss thus corresponding to lipothymia, which is not taken into account by the FHHA definition. Median length of stay in the ED was 4 hours and 67 patients $(22,6 \%)$ were hospitalized. Minimal estimated cost was 280000 euros. Risk factors independently associated with hospitalization were age $\geq 60$ years-old and complete consciousness loss unlike predisposing circumstances to vagal hypertonia.

Conclusion: Age $\geq 60$ years-old and complete consciousness loss seemed to be associated with hospitalization. 


\section{1)Introduction :}

Le «malaise » est un motif de consultation extrêmement fréquent au sein des services d'accueil des urgences (SAU) (3 à 10\% selon les études) [1-3].

L'origine étymologique du terme malaise signifie «état de mal-être, où l'on est pas à son aise ». Ce symptôme subjectif peut être lié à des processus physiopathologiques très différents (figure 1) allant d'affections bénignes dues à une hypertonie parasympathique à des cardiopathies graves mettant le pronostic vital en jeu à court terme (exemple : bloc auriculoventriculaire de haut degré). Classiquement, les malaises correspondent à un épisode aigu caractérisé par un trouble de la conscience ou de la vigilance plus ou moins complet, spontanément régressif et sont différenciés en syncopes, lipothymies ou pertes de connaissance brèves en fonction de la vitesse d'installation et de la profondeur de l'altération de la vigilance [1] (tableau 1). Des recommandations ont été élaborées sur la base de ces définitions par la Société Française de Médecine d’Urgence [2,3] à partir d'une bibliographie exclusivement d'origine anglo-saxonne.

Depuis, de nouvelles recommandations ont été publiées par «l'european society of cardiology » [4] puis traduites par la Haute autorité de santé (HAS) [5]. Ces recommandations evidence-based ne concernent que les syncopes définies ici par : «une perte de connaissance de début rapide, de durée généralement brève, spontanément résolutive, s'accompagnant d'une perte de tonus postural avec un retour rapide à un état de conscience normale et due à une hypoperfusion cérébrale globale et passagère » [4-5]. Cette définition ne recoupe pas du tout les mêmes réalités que celles balayées par le symptôme «malaise » distinguant les syncopes, les lipothymies ou les pertes de connaissance brèves et elle impose de connaître le mécanisme du malaise avant de savoir si ces dernières recommandations sont applicables.

Actuellement, les SAU sont le recours de premier soin de nombreux patients et les malaises y sont très fréquemment systématiquement référés [1,6]. On demande de nos jours de plus en plus aux SAU dont l'organisation est mise à rude épreuve et dont tout dysfonctionnement est rapidement dénoncé par les médias [7]. Ceci est d'autant plus difficile que la prise en charge des malaises ou même des syncopes selon la définition anglo-saxonne reprise par l'HAS n'est pas standardisée, qu'elle repose plus sur le jugement clinique au cas par cas que sur des consensus établis et que les hospitalisations pour surveillance qui peuvent en découler sont jugées inutilement coûteuses [6, 8-13].

Devant l'absence de données épidémiologiques françaises récentes sur les malaises, nous avons mené une étude épidémiologique descriptive et analytique pour apprécier (i) le 
poids des malaises en terme de consultations au sein d'un service d'accueil des urgences d'un centre hospitalier universitaire (CHU) sur une durée de 3 mois, (ii) l'épidémiologie clinique et (iii) le cout médico-économique des malaises (temps de passage aux urgences, biologie, hospitalisation) en s'intéressant tout particulièrement aux facteurs associés à l'hospitalisation. 


\section{2)Matériel et Méthodes :}

2.1) Type d'étude: Etude épidémiologique descriptive et analytique, rétrospective monocentrique, conduite à partir des données issues du logiciel Urqual® du SAU du CHU de Reims, hôpital de 1100 lits pour un bassin de population d'environ 300000 habitants.

2.2) Critères d'inclusion: Patients âgés de plus de 15 ans ayant consulté au Service d'accueil des urgences médicales adulte du CHU de Reims, entre le $1^{\text {er }}$ janvier 2012 et le 31 mars 2012 pour le motif «malaise » inscrit comme tel par l'infirmière d'accueil et d'orientation (IAO) sur la main courante, qu'ils consultent d'eux même ou soient référés par un médecin traitant ou les sapeurs pompiers.

N'ont pas été inclus dans l'étude:

- les patients pour lesquels l'interrogatoire médical rapporté dans le dossier a permis de retrouver un autre motif de consultation que malaise (douleurs thoraciques, palpitations, dyspnée, déficits neurologiques transitoires, syndrome anémique ou vertiges)

- les malaises authentiques dans un contexte fébrile ou d'intoxication (alcool ou médicamenteuse)

- les chutes chez les personnes âgées renseignées comme telles dans la main courante et celles renseignées comme malaise mais pour lesquelles l'interrogatoire médical rapporté dans le dossier a permis de retrouver l'absence de trouble de vigilance résolutif.

2.3) Critères d'exclusion: Ont été exclus de l'analyse de l'épidémiologie clinique et du devenir immédiat des malaises :

- les patients partis sans être vus par un médecin, sortis contre avis médical ou ayant fugué.

- les patients pour lesquels l'observation clinique était incomplète (pas de description du malaise en termes de prodromes, vitesse d'installation et profondeur de la perte de connaissance).

Les patients ayant des malaises à répétition dans la période de l'étude n'ont été inclus qu'une seule fois à l'occasion du premier malaise. 
2.4) Variables recueillies : les données suivantes ont été recueillies pour chaque patient et colligées grâce au tableur Excel® Microsoft :

- Sexe et âge du patient lors de la consultation

- Antécédents de cardiopathie ou de pathologie neurologique potentiellement épileptogène

- Traitements actuels par hypoglycémiant, antihypertenseur, psychotrope dont antiépileptique

- Temps passé aux urgences en heures

- Description du malaise : circonstances favorisant l'hypertonie vagale (lieu confiné, chaleur, émotion, repas, période post-prandiale, miction, défécation, après un effort) [5], prodromes en faveur d'un début progressif (sueurs, palpitations, faiblesse des jambes, sensation de tête vide), perte de connaissance complète ou non, pâleur, morsure de langue, perte d'urine ou mouvements anormaux per critiques, confusion ou asthénie post critique, présence d'une douleur thoracique associée

- Données de l'examen clinique : bradycardie (pouls < à 60bpm), pression artérielle et recherche d'une hypotension orthostatique définie par une perte de $20 \mathrm{~mm} \mathrm{Hg}$ de la pression artérielle systolique ou $10 \mathrm{~mm} \mathrm{Hg}$ de la pression artérielle diastolique en position debout. L'examen clinique était considéré comme normal en l'absence de pâleur, de mouvements anormaux, de perte d'urines, de morsure de langue, de bradycardie, d'hypotension orthostatique quand elle était recherchée et de symptômes post-critiques tels qu'une confusion mentale.

- Examens complémentaires effectués : électrocardiogramme (ECG), glycémie capillaire, biologie, avis spécialisé

- Devenir du patient : retour à domicile ou hospitalisation et durée d'hospitalisation en jours

- Diagnostic étiologique final retenu au décours de la prise en charge médicale aux urgences ou en hospitalisation.

2.5) Analyse rétrospective des données : Tous les dossiers ont été revus consensuellement par deux reviewers externes (EG et YN) et reclassés selon les 2 classifications classique [1] et de la Haute Autorité de Santé [5].

2.6) Coût médico-économique des malaises: Le coût financier minimal a été calculé en multipliant le nombre de consultations aux urgences sur la période de l'étude par le coût 
minimal d'une consultation aux urgences (sans majoration de nuit ou pour biologie) et en multipliant le nombre d'hospitalisations sur la période de l'étude par la durée médiane d'hospitalisation (jours) et par le coût minimal d'une journée d'hospitalisation en médecine.

2.7) Analyse statistique: Les variables qualitatives exprimées en pourcentage ont été comparées grâce au test du Khi deux si applicable, ou à défaut grâce au test exact de Fischer. Les variables quantitatives ont été comparées grâce au test de Mann Whitney. Un p <0,05 était considéré comme significatif. Les variables d'intérêt avec un $p<0,05$ en analyse univariée ont été rentrées dans un modèle de régression logistique multivarié pas à pas. L'analyse a été réalisée grâce aux logiciels Statview® et SAS® version 9.3 (SAS Institute Inc., Cary, North Carolina, USA).

2.8) Accord CNIL : Un accord CNIL n'a pas été nécessaire du fait de l'absence de collection de données autres que celles déjà présentes sur le réseau informatique du CHU. 


\section{3) Résultats :}

3.1) Poids des malaises en terme de consultations au sein du SAU :

Entre le $1^{\text {ier }}$ janvier 2012 et le 31 mars 2012, 341 patients (5,7\%) parmi les 5953 patients qui ont consulté aux urgences médicales durant cette période, correspondaient aux critères d'inclusion (figure 2).

\section{2) Epidémiologie clinique des malaises:}

3.2.1) caractéristiques de la population :

Quarante cinq patients parmi les 341 (13,2\%) ont été exclus de l'analyse du fait de données insuffisantes dans le dossier médical ou du fait d'un départ sans être examinés (fugue, sortie contre avis médical). Seuls 296 (86,8\%) patients avaient des données suffisantes pour être analysées (figure 2).

La population étudiée comprenait une majorité de femmes (62,8\%). L’âge médian des patients inclus était de 43 ans avec des extrêmes de 15 à 93 ans. La présence d'une cardiopathie à l'interrogatoire n'était retrouvée que dans 15,8\% des cas. Les données démographiques et médicales concernant les 296 patients inclus sont présentées dans le tableau 2.

Le tableau 3 présente les caractéristiques cliniques des malaises ainsi que la prise en charge médicale en rapport. Des prodromes étaient retrouvés dans 76,0\% des cas et la perte de connaissance était complète dans $52,0 \%$ des cas. L'examen clinique était normal dans $57,1 \%$ des cas. Le test d'hypotension orthostatique et un ECG étaient effectués aux urgences dans $13,8 \%$ et $83,1 \%$ des cas respectivement. Soixante sept patients $(22,6 \%)$ ont été hospitalisés en service de médecine pour une durée médiane de 5 jours.

\subsection{2) classification et causes des malaises:}

Les malaises ont été reclassés selon la définition classique [1] en lipothymies, pertes de connaissance brèves et syncopes (tableau 1) dans 142, 106 et 48 cas respectivement.

Les causes retrouvées parmi les lipothymies, pertes de connaissance brèves et syncopes sont présentées dans les tableaux 4,5 et 6 . 
Les 3 causes les plus fréquentes parmi les 142 lipothymies étaient vagales, psychogènes, et par hypotension orthostatique. Elles représentaient $84,5 \%$ des causes de lipothymies dans cette série.

Les 3 causes les plus fréquentes parmi les 106 pertes de connaissance brèves étaient vagales, épileptiques et hypoglycémiques. Elles représentaient $80,2 \%$ des causes de pertes de connaissance brèves dans cette série.

Les 3 causes les plus fréquentes parmi les 48 syncopes étaient vagales, indéterminées et cardiogéniques. Elles représentaient 89,6\% des causes de syncopes dans cette série.

La cause réflexe par hypertonie vagale était la principale cause de malaise retrouvée grâce à cette classification, correspondant à 151 des 296 malaises (51,0\%).

Seuls 97 des 296 malaises (32,7\%) correspondaient aux syncopes selon la définition reprise par l'HAS [5], ils correspondaient aux 48 syncopes selon la définition classique [1] associées à 49 des pertes de connaissance brèves.

Les causes retrouvées parmi les syncopes selon la définition reprise par l'HAS sont présentées dans le tableau 7. Les 3 causes les plus fréquentes étaient vagales, cardiogéniques et indéterminées. Elles représentaient $92,8 \%$ des causes des syncopes selon la définition reprise par l'HAS dans cette série.

Parmi les 67 patients hospitalisés, on retrouvait 13 syncopes, 16 lipothymies et 38 pertes de connaissance brèves. Le taux d'hospitalisation des patients ayant une perte de connaissance brève (38 sur $106 ; 35,8 \%$ ) était statistiquement supérieur au taux d'hospitalisation des patients souffrant d'autres types de malaises (29 sur $190 ; 15,2 \%$ ) $(\mathrm{p}<0,0001)$. Les diagnostics en sortie d'hospitalisation ne différaient de ceux établis aux urgences que dans 14 cas parmi les 67 patients hospitalisés (20,9\%). De manière intéressante, parmi ces 14 cas de malaises, $5(35,7 \%)$ étaient liés à une hypotension orthostatique. Aucun patient n'est décédé durant son hospitalisation $(n=67)$ ou durant son passage exclusif aux urgences $(n=229)$.

\section{3) Coût médico-économique des malaises}

\subsection{1) temps passé aux urgences:}

Les 296 patients sont restés une durée médiane de 4 heures [0,5-15] aux urgences notamment car une biologie était réalisée dans $77,7 \%$ des cas et un avis spécialisé était sollicité dans $26,3 \%$ des cas (tableau 3). Les patients hospitalisés (n=67) sont restés un temps 
médian de 6 [1,5-14] heures alors que les patients non hospitalisés $(\mathrm{n}=229)$ sont restés un temps médian de $4[0,5-15]$ heures $(\mathrm{p}<0,0001)$.

\subsection{2) coût financier :}

Durant les 3 mois de l'étude, 229 patients n'ont effectué qu'une consultation aux urgences tandis que 67 ont été hospitalisés pour une durée médiane de 5 jours (tableau 3). Le coût de ces hospitalisations correspondait à un coût minimal de 67 X5X800 $=268000$ euros soit 95,7\% des 280000 euros (268 000+229X48=268 000+11 000) correspondant au coût des consultations et des hospitalisations de l'ensemble des patients pris en charge pour malaise sur la période de l'étude.

\subsection{3) variables associées à l'hospitalisation}

Indépendamment du coût, nous nous sommes ensuite interrogés sur le profil des patients hospitalisés afin de savoir si l'on pouvait cibler directement les patients à hospitaliser afin d'éviter un surencombrement par accumulation de patients en attente aux urgences.

Les variables statistiquement associées à l'hospitalisation sont présentées dans le tableau 8. L'âge, le sexe masculin, la présence d'une cardiopathie, d'une épilepsie, d'un traitement antihypertenseur, hypoglycémiant, antiépileptique ou psychotrope et d'une perte de connaissance complète étaient statistiquement associés à l'hospitalisation en analyse univariée. La présence de prodromes, circonstances favorisantes ou d'un examen clinique normal était associée à l'absence d'hospitalisation en analyse univariée.

Les variables significatives en analyse univariée ont été rentrées dans un modèle multivarié dont les résultats sont présentés dans le tableau 9. Seuls l'âge et la perte de connaissance complète étaient indépendamment associés à l'hospitalisation. La présence de circonstances favorisantes était indépendamment protectrice de l'hospitalisation alors que l'examen clinique normal ne l'était pas. Le sexe n'était plus significativement associé à l'hospitalisation une fois que les circonstances favorisantes et la perte de connaissance complète étaient incluses dans le modèle multivarié tout comme la présence d'une cardiopathie, d'une épilepsie ou d'un traitement antihypertenseur, hypoglycémiant, antiépileptique ou psychotrope n'étaient plus significativement associés à l'hospitalisation une fois que l'âge était inclus dans le modèle multivarié. Des résultats identiques étaient observés lorsque la variable qualitative âge $\geq 60$ ans (Odds ratio 4,7, IC95\% 2,4-9,0, p<0,0001) était rentrée dans le modèle multivarié (données non montrées). 


\section{4)Discussion :}

Ce travail illustre que les malaises correspondaient à 5,7\% des consultations durant le $1^{\text {er }}$ trimestre 2012 au sein du SAU du CHU de Reims. La population concernée était mixte avec une discrète prédominance féminine et âgée de 15 à 93 ans (médiane 43 ans) dans notre étude. Dans $48 \%$ des cas évaluables $(n=142)$, il n'y avait pas de perte de connaissance complète et le malaise correspondait donc à une lipothymie non prise en compte dans la classification valorisée par l'HAS. L'examen clinique était normal dans 57\% des cas. Les patients restaient une durée médiane de 4 heures aux urgences, alors que seuls $22,6 \%$ d'entre eux étaient hospitalisés. En dehors du coût financier, nous nous sommes interrogés dans une problématique d'occupation du SAU, s'il ne fallait pas envisager une sélection en amont des malades à référer aux urgences ou à hospitaliser directement. Les facteurs de risque indépendamment associés à une hospitalisation dans cette étude étaient un âge $\geq 60$ ans ou la présence d'une perte de connaissance alors que la présence de circonstances favorisant l'hypertonie vagale était un événement protecteur de l'hospitalisation.

\section{1) Poids des malaises en terme de consultations au sein du $S A U$ :}

Le «malaise» est un motif de consultation fréquent correspondant à 5,7\% des consultations au sein du SAU du CHU de Reims. Cela est cohérent avec les données de la littérature française et européenne, où l'on trouve des fréquences estimées entre 3 et $15 \%$ des motifs de consultation [1-3,14]. Un chiffre analogue de 5,3\% est rapporté dans le travail de Geering et collègues concernant les consultations au sein du SAU de Neuchâtel en 2009 [15]. Toutefois, la comparaison des résultats de cette étude avec les séries publiées à partir d'autres services d'accueil des urgences peut être rendue difficile par l'utilisation de critères d'inclusion différents des nôtres. En effet, nos critères étaient relativement sévères afin d'exclure de l'analyse tous les autres motifs de consultation proches du malaise (vertige, syndrome confusionnel, chute...). De plus, la comparaison aux études anglo-saxonnes est limitée car le terme «malaise » n'y existe pas. Dans ces études où seules les syncopes sont prises en compte, ces dernières ne représentent qu'1 à 3\% des consultations dans un service d'urgence [4-6,15], ce qui est assez proche du chiffre de $1,6 \%$ retrouvé dans ce travail. Par contre, il n'est pas exclu qu'il existe de profondes variations géographiques ou saisonnières de l'épidémiologie des malaises, non évaluables par cette étude hivernale et monocentrique. 


\section{2) Epidémiologie clinique des malaises:}

Les patients de notre série ayant consulté au SAU pour malaise durant la période de l'étude étaient plutôt jeunes (âge médian de 43 ans) et étaient préférentiellement des femmes. Ces résultats sont également retrouvés dans les principales séries de la littérature bien que ces dernières soient anciennes [14] ou de méthodologies différentes [16,17]. Souvent, il s'agissait de patients sans comorbidité ni traitement (tableau 2). Dans $48 \%$ des cas évaluables ( $n=142)$, il n'y avait pas de perte de connaissance et le malaise correspondait donc à une lipothymie ou near syncope non prise en compte dans les recommandations elassifieation-anglo-saxonnes reprises par 1'HAS [4,5]. Les syncopes elassées grâce à définies selon cette classification [5] ne rendent compte que de 97 des 296 patients de cette série, ce qui signifie que pour 67,2\% des malaises (199 sur 296), les recommandations HAS n'aident en rien à la prise en charge des patients. Toutes ces données suggèrent que (i) les malaises dans cette étude semblent relativement bénins et que (ii) l'ancienne classification en syncope, lipothymie et perte de connaissance brève grâce à l'interrogatoire $[1,18]$ semble plus intéressante pour apprécier la gravité des patients référés pour malaise que la classification HAS dans la pratique de tous les jours. Même dans la bibliographie anglo-saxonne limitée aux syncopes, le jugement clinique au cas par cas basé sur les données de l'examen clinique et de l'interrogatoire n'est pas inférieur à tous les scores prédictifs de gravité [8-11].

De manière intéressante, on s'aperçoit que la recherche d'hypotension orthostatique et l'ECG n'étaient réalisés que dans $14 \%$ et $83 \%$ des cas respectivement alors que ces examens recommandés en première intention devant un malaise auraient dû être réalisés dans $100 \%$ des tous les cas $[1,3,5]$. Ces données sont retrouvées dans la thèse d'exercice de Pierre VarletAndré à Dijon [16]. Cela semble d'autant plus dommage que (i) l'hypotension orthostatique est dans la littérature un diagnostic fréquent représentant 6 à 23\% des étiologies de malaise [7] (ii) que ce test peu coûteux aurait peut-être permis ici d'éviter ou d'écourter 5 hospitalisations dans notre étude. Đans la littérature, Même si la rentabilité de l'ECG post-critique n'est que de 5\%, mais la gravité et la surmortalité des causes cardiaques justifient sa réalisation systématique $[1,2]$.

\section{3) Coût médico-économique des malaises}

La vraie problématique aux urgences peut apparaître en fait comme la difficulté de trier les patients à risque vital à court terme de la masse de malaises bénins principalement par origine réflexe par hypertonie vagale. Dans ce contexte, les malades restaient une durée médiane de 4 heures aux urgences, souvent dans l'attente d'une biologie ou d'un avis 
spécialisé dans $77,7 \%$ et $26,3 \%$ des cas respectivement. Cette démarche chronophage est également coûteuse financièrement avec des coûts minimaux estimés à 11000 et 268000 euros respectivement pour les 229 consultations et 67 hospitalisations pour malaise sur les 3 mois de l'étude. pour l'ensemble des consultations aboutissant à une hospitalisation dans semlement 22.6\% des ( $\mathrm{n}=67$ ). Le taux d'hospitalisation de $22.6 \%$ de cette série tenant compte des malaises sans perte de connaissance complète comme les lipothymies est logiquement inférieur au taux d'hospitalisation minimal rapporté (30\%) dans les études dédiées exclusivement aux syncopes [6,17,19]. Si l'on considère les hospitalisations qui correspondent à 95,7\% du coût total correspondant à la prise en charge des patients de cette étude, le coût financier des malaises s'élève, au minimum, à 268000 euros sur les 3 mois de l'étude, est de près de 4000 euros par patient hospitalisé. Ce chiffre semble cohérent avec ceux les coûts moyens- de 3506 +/- 2729 euros et 5400 dollars par hospitalisation rapportés dans des études italienne [19] et américaine [13] respectivement évalué qui-évalue ce coût à par pour les patients hospitalisés. De nombreuses stratégies de réduction de coût sont en cours d'évaluation car les hospitalisations pour le diagnostic de syncopes selon la définition anglosaxonne sont très régulièrement non prises en charge par les systèmes de protection sociale gouvernementaux américains Medicare et Medicaid [6,12]

Le design de cette étude ne permet pas de juger la pertinence des hospitalisations, vu l'absence de données ultérieures recueillies permettant d'évaluer la bénignité ou non des malaises hospitalisés et une éventuelle utilité/futilité de l'hospitalisation. Pour ce faire, il aurait fallu récolter des données de suivi supplémentaires auprès des médecins traitants sous couvert d'un accord CNIL. Nous sommes donc partis du postulat que toutes les hospitalisations étaient adaptées et avons négligé les hospitalisations réalisées parfois en unité de surveillance temporaire du fait de l'incapacité à faire repartir les patients à leur domicile la nuit (données personnelles non publiées). Nous avons voulu savoir quel était le profil des patients hospitalisés,--plus dans une logique de prévention de d'épargne d'surencombrement des urgences que financière, car les patients hospitalisés restaient statistiquement plus longtemps aux urgences que les patients non hospitalisés $(\mathrm{p}<0.0001)$. La classification en syncope/lipothymie/perte de connaissance brève n'apparaît pas être une aide de prime abord car tous ces sous types de malaise sont représentés parmi les patients hospitalisés. Toutefois, en s'aperçoit que le taux d'hospitalisation statistiquement supérieur $(\mathrm{p}<0.0001)$ des patients ayant une perte de connaissance brève par rapport aux patients souffrant d'autres types de malaises les pertes de connaissance brèves sont surreprésentées parmi les hospitalisés par rapport aux autres types de malaises selon la classification classique [1], suggère que et nous 
interprétons alors l'hospitalisation pour surveillance est la seule alternative face au doute du clinicien par rapport au risque de morbidité/mortalité à court terme du patient devant un malaise de mécanisme mal appréhendé et classé dans la catégorie perte de connaissance brève dont la définition est la plus large (tableau 1). L'analyse statistique des autres données médicales ou démographiques a été menée afin de savoir quels éléments étaient utilisés par le clinicien pour pondérer son doute et déclencher une hospitalisation. Les facteurs de risque indépendamment associés à une hospitalisation dans notre étude étaient l'âge en variable quantitative ou qualitative $\geq 60$ ans ou la présence d'une perte de connaissance complète alors que la présence de circonstances favorisant les malaises réflexes par hypertonie vagale était un événement protecteur de l'hospitalisation. Le cut-off à 60 ans, a été défini ici de manière arbitraire dans l'optique d'avoir une variable qualitative plus pratique à utiliser dans la vie de tous les jours que la variable quantitative plus puissante statistiquement. Ce cut-off est surtout basé sur les âges moyens des patients hospitalisés ou non hospitalisés (tableau 8) et doit être revalidé dans une ou d'autres cohortes indépendantes. Si tel est le cas, ces données épidémiologiques suggéreraient que l'on puisse potentiellement désencombrer les urgences en ne référant que les patients âgés de plus de 60 ans et/ou avec une perte de connaissance complète voire en les hospitalisant directement dans une unité dédiée «syncope unit » comme cela est évoqué dans les recommandations européennes pour la prise en charge des syncopes $[4,20]$. Les malaises avec circonstances favorisantes ou sans perte de connaissance complète survenant chez un patient âgé de moins de 60 ans pourraient être gérés en médecine de ville (généraliste, maison médicale, SOS médecin...) avec ou sans l'aide de la télémédecine pour l'interprétation de l'ECG. Il convient bien évidemment de valider d'abord ces données dans une ou d'autres cohortes indépendantes avant d'envisager la réalisation d'une étude expérimentale prospective d'évaluation diagnostique, seul type d'étude à même de valider une stratégie médico-économique populationnelle. En effet, une telle stratégie de stratification du risque pourrait s'avérer plus aisée dans l'ensemble des malaises que dans les syncopes seules [11], mais la validation dans une autre cohorte indépendante est nécessaire car notre étude souffre bien évidemment de biais :

- biais de sélection des patients (petite agglomération, période hivernale, et caractère possiblement subjectif du diagnostic de malaise retenu par l'IAO)

- biais de classement des données du fait du recueil rétrospectif à partir des données des dossiers des urgences dont la qualité n'était pas constante. Par contre, nous espérons que la revue des dossiers par deux reviewers externes corrige la subjectivité que l'on 
peut avoir à la lecture d'un dossier sans qu'un rapport de hiérarchie ne s'installe entre les 2 reviewers.

- et surtout un biais d'interprétation de la bénignité apparente des malaises, basée sur l'âge, les comorbidités et la présentation clinique du malaise, du fait de l'absence de données pronostiques ultérieures, raison pour laquelle nous ne nous sommes pas permis de juger la pertinence des hospitalisations (cf supra).

En conclusion, les données de cette étude suggèrent que la classification classique des malaises en syncope, lipothymie et perte de connaissance brève reste d'intérêt pour l'évaluation des malaises qui reste un motif de consultation fréquent (5\%) d'une population mixte et de tout âge au sein des SAU. La présence d'une lipothymie, sans perte de connaissance est retrouvée dans un cas sur deux et associée le plus souvent à des étiologies bénignes. L'examen clinique y est normal le plus souvent mais un électrocardiogramme et une recherche d'hypotension orthostatique doivent être systématiquement réalisés. Un âge supérieur ou égal à 60 ans et/ou la présence d'une perte de connaissance complète sont associés statistiquement à l'hospitalisation, à l'inverse de la présence de circonstances favorisant les malaises réflexes par hypertonie vagale. Ces éléments pourraient servir à trier les malades en amont des urgences, afin d'éviter un surencombrement des structures d'accueil, mais ceci doit être validé dans d'autres cohortes indépendantes avant d'envisager une étude expérimentale prospective d'évaluation diagnostique.

\section{Remerciements: Maryvonne Sartelet, Dr Carole Carolet.}

Conflit d'intérêts : aucun.

Source de financement : aucune 


\section{Références:}

[1] Lestavel P, Halle I, Forget AP. Conduite à tenir devant un malaise, une syncope et une perte de connaissance. JEUR 1997; $4:$ 192-6. http://uhcd.chra.free.fr/procedures/cardio/malaises_jeur.pdf. Accès au site le 08/09/2015.

[2] Société Française de Médecine d’Urgence ; VIème Conférence de Consensus. Prise en charge des malaises au service d'accueil des urgences. Conférence de consensus, Grenoble, Alpes Congrès, 1996. http://www.sfmu.org/upload/consensus/cc_malaises.pdf. Accès au site le $08 / 09 / 2015$.

[3] Le Gall C, Philippe JM, Busseuil C, Carpentier F, Leuret A, Sommereisen JP et al. Actualisation de la VIème Conférence de Consensus de 1996. Société Française de Médecine d'Urgence ; Commission de veille 2005. http://www.sfmu.org/upload/consensus/Actualisation_Malaise.pdf. Accès au site le 08/09/2015.

[4] Moya A, Sutton R, Ammirati F, Blanc JJ, Brignole M, Dahm JB, et al. Guidelines for the diagnosis and the management of syncope (version 2009). The Task Force for the Diagnosis and Management of Syncope of the European Society of Cardiology (ESC). Eur. Heart J 2009; 30: 2631-71.

[5] Collège de l'HAS. Recommandations professionnelles : Pertes de connaissance brèves de l'adulte : prise en charge diagnostique et thérapeutique des syncopes, mai 2008.

http://www.has-sante.fr/portail/jcms/c_681730/syncopes-recommandations. Accès au site le $08 / 09 / 2015$.

[6] Grossman AM, Volz KA, Shapiro NI, Salem R, Sanchez LD, Smulowitz P, et al. Comparison of 1-Day Emergency Department Observation and Inpatient Ward for 1-Day Admissions in Syncope Patients. J Emerg Med. 2016; 50: 217-22.

[7] Décès à Cochin : des « dysfonctionnements » aux urgences selon l'enquête. Le Parisien.fr. 2014 Fév. http://www.leparisien.fr/faits-divers/deces-a-cochin-des-dysfonctionnements-auxurgences-selon-1-enquete-28-02-2014-3631507.php. Accès au site le 08/09/2015. 
[8] Williamson C, Reed MJ. Syncope: the emergency department and beyond. Intern Emerg Med. 2015; 10: 843-50

[9] Reed MJ, Newby DE, Coull AJ, Prescott RJ, Jacques KG, Gray AJ. The ROSE (risk stratification of syncope in the emergency department) study. J Am Coll Cardiol. 2010; 55: 713-21

[10] Costantino G, Casazza G, Reed M, Bossi I, Sun B, Del Rosso A, et al. Syncope risk stratification tools vs clinical judgment: an individual patient data meta-analysis. Am J Med. 2014; 127: 1126.e13-25.

[11] Costantino G, Sun BC, Barbic F, Bossi I, Casazza G, Dipaola F, et al. Syncope clinical management in the emergency department: a consensus from the first international workshop on syncope risk stratification in the emergency department. Eur Heart J. 2016; 37: 1493-8.

[12] Lin M, Wolfe RE, Shapiro NI, Novack V, Lior Y, Grossman SA. Observation vs admission in syncope: can we predict short length of stays? Am J Emerg Med. 2015; 33:16846.

[13] Sun BC, Emond JA, Camargo CA Jr. Direct medical costs of syncope-related hospitalizations in the United States. Am J Cardiol. 2005; 95:668-71.

[14] Berthier F, Soulat LC, Girard JJ, Parisot R, Baron D. Malaises et pertes de connaissance au service d'urgence : incidence et exactitude du diagnostic. Réan Urg 1997 ; 6 : 443-8. http://www.sciencedirect.com/science/article/pii/S1164675697801405. Accès au site le 08/09/2015.

[15] Geering S, Hanhart W, Kehtari R. Quand référer aux urgences un patient présentant un malaise? Rev Med Suisse 2010 ; 6 :1535-39.

[16] Varlet-André P. Etude épidémiologique de 205 patients admis pour "malaise" aux urgences du centre hospitalier universitaire de Dijon. [Thèse]. Dijon : Faculté de médecine de Dijon ; 2013. 
[17] Sun BC, Emond JA, Camargo CA Jr. Characteristics and admission patterns of patients presenting with syncope to U.S. emergency departments, 1992-2000. Acad Emerg Med. 2004;11: 1029-34.

[18] Blettery B, Corgibert-Escalier F, Virot C, Tavernier C. Les malaises dans un service d'urgence. Approche diagnostique. Presse Med 1989; 18 :753-6.

[19] Brignole M, Ungar A, Bartoletti A, Ponassi I, Lagi A, Mussi C et al. Standardized-care pathway vs. usual management of syncope patients presenting as emergencies at general hospitals. Europace. 2006; $8: 644-50$.

[20] Moser C, Salort A. Prise en charge des syncopes aux urgences: un nouvel outil pour une meilleure orientation? [Thèse]. Grenoble : Université Joseph Fourier, Faculté de médecine de Grenoble ; 2012. 
Tableaux:

Tableau 1. Résumé des principales caractéristiques des «malaises » selon la classification Syncope, Lipothymie, Perte de connaissance brève d'après [1].

\begin{tabular}{|l|l|l|l|}
\hline \multicolumn{4}{|c|}{ «Malaise » } \\
\hline Caractéristiques & Syncope & Lipothymie & Perte de connaissance brève \\
\hline Début & Brutal & Progressif & Progressif \\
\hline Perte de conscience complète & Oui & Non & Oui \\
\hline $\begin{array}{l}\text { Retour rapide à un état de } \\
\text { conscience normale }\end{array}$ & Oui & Oui & Variable \\
\hline
\end{tabular}


Tableau 2. Caractéristiques démographiques et médicales des 296 patients inclus.

\begin{tabular}{|l|c|}
\hline & Population incluse $\mathrm{n}=296(100 \%)$ \\
\hline Sexe féminin $\mathrm{n}=(\%)$ & $186(62,8 \%)$ \\
\hline Age (années) médiane [extrêmes] & $43[15-93]$ \\
\hline Antécédents de cardiopathie $\mathrm{n}=(\%)$ & $47(15,8 \%)$ \\
\hline Antécédents de pathologie neurologique $\mathrm{n}=(\%)$ & $21(7,1 \%)$ \\
\hline Traitement actuel par hypoglycémiants $\mathrm{n}=(\%)$ & $37(12,5 \%)$ \\
\hline Traitement actuel par antihypertenseurs $\mathrm{n}=(\%)$ & $81(27,3 \%)$ \\
\hline Traitement actuel par psychotropes $\mathrm{n}=(\%)$ & $56(18,9 \%)$ \\
\hline
\end{tabular}


Tableau 3. Caractéristiques cliniques des malaises et description de la prise en charge médicale des 296 patients inclus.

\begin{tabular}{|c|c|c|}
\hline & $\begin{array}{l}\text { Population incluse } n=296 \\
\qquad(100 \%)\end{array}$ & Données manquantes \\
\hline Circonstances favorisant malaise $\mathrm{n}=(\%)$ & $160(54,0 \%)$ & 12 \\
\hline Prodromes $n=(\%)$ & $225(76,0 \%)$ & 0 \\
\hline- sueurs $n=(\%)$ & $95(32,1 \%)$ & 0 \\
\hline - palpitations $\mathrm{n}=(\%)$ & $42(14,2 \%)$ & 0 \\
\hline - autres* $\mathrm{n}=(\%)$ & $109(48,4 \%)$ & 0 \\
\hline Perte de connaissance complète $n=(\%)$ & $154(52,0 \%)$ & 0 \\
\hline Pâleur per critique $n=(\%)$ & $23(7,8 \%)$ & 0 \\
\hline Mouvements anormaux $\mathrm{n}=(\%)$ & $36(12,2 \%)$ & 0 \\
\hline Perte d'urine $\mathrm{n}=(\%)$ & $13(4,4 \%)$ & 0 \\
\hline Morsure de langue $n=(\%)$ & $13(4,4 \%)$ & 0 \\
\hline Confusion post critique $n=(\%)$ & $40(13,5 \%)$ & 0 \\
\hline Asthénie post critique $n=(\%)$ & $43(14,5 \%)$ & 0 \\
\hline Présence bradycardie $\mathrm{n}=(\%)$ & $36(12,2 \%)$ & 17 \\
\hline Douleur thoracique $\mathrm{n}=(\%)$ & $22(7,4 \%)$ & 0 \\
\hline $\begin{array}{l}\text { Valeur de la pression artérielle (systolique moyenne } \\
\pm \text { écart type/diastolique moyenne } \pm \text { écart type) } \\
(\mathrm{mmHg})\end{array}$ & $130,2 \pm 22,6 / 71,9 \pm 12,2$ & 24 \\
\hline Test hypotension orthostatique réalisé $\mathrm{n}=(\%)$ & $41(13,8 \%)$ & 0 \\
\hline Présence d'une hypotension orthostatique $n=(\%)$ & $7(2,4 \%)$ & 0 \\
\hline Examen clinique normal $\mathrm{n}=(\%)$ & $169(57,1 \%)$ & 0 \\
\hline Electrocardiogramme réalisé $n=(\%)$ & $246(83,1 \%)$ & 0 \\
\hline Glycémie capillaire réalisée au SAU n= (\%) & $218(73,6 \%)$ & 0 \\
\hline Biologie réalisée $\mathrm{n}=(\%)$ & $230(77,7 \%)$ & 0 \\
\hline Avis spécialisé au SAU n= (\%) & $78(26,3 \%)$ & 0 \\
\hline Temps passé au SAU ${ }^{+}$(heures) médiane [extrêmes] & $4[0,5-15]$ & 0 \\
\hline Hospitalisation $\mathrm{n}=(\%)$ & $67(22,6 \%)$ & 0 \\
\hline Durée d'hospitalisation (jours) médiane [extrêmes] & $5[1-45]$ & 0 \\
\hline
\end{tabular}

* faiblesse des membres inférieurs, sensation de tête vide et prodromes non détaillés dans l'observation. ${ }^{+}$SAU : service d'accueil des urgences. 
Tableau 4. Causes des lipothymies définies selon la classification classique [1].

\begin{tabular}{|l|c|}
\hline & Lipothymies $\mathrm{n}=142(100 \%)$ \\
\hline Origine réflexe dont vagale $\mathbf{n}=\mathbf{( \% )}$ & $\mathbf{7 8}(\mathbf{5 4 , 9 \% )}$ \\
\hline Psychogène $\mathbf{n}=\mathbf{( \% )}$ & $\mathbf{2 7}(\mathbf{1 9 , 0 \% )}$ \\
\hline Hypotension orthostatique $\mathbf{n}=\mathbf{( \% )}$ & $\mathbf{1 5}(\mathbf{1 0 , 6 \% )}$ \\
\hline Indéterminée $\mathrm{n}=(\%)$ & $11(7,8 \%)$ \\
\hline Hypoglycémie $\mathrm{n}=(\%)$ & $8(5,6 \%)$ \\
\hline Cardiopathie $\mathrm{n}=(\%)$ & $2(1,4 \%)$ \\
\hline Epilepsie ou crise convulsive $\mathrm{n}=(\%)$ & $1(0,7 \%)$ \\
\hline
\end{tabular}

En gras, les 3 causes les plus fréquentes. 
Tableau 5. Causes des pertes de connaissance brèves définies selon la classification classique [1].

\begin{tabular}{|l|c|}
\hline & Pertes de connaissance brèves $\mathrm{n}=106(100 \%)$ \\
\hline Origine réflexe dont vagale $\mathbf{n}=\mathbf{( \% )}$ & $\mathbf{4 5}(\mathbf{4 2 , 5 \% )}$ \\
\hline Epilepsie ou crise convulsive $\mathbf{n}=\mathbf{( \% )}$ & $\mathbf{3 0}(\mathbf{2 8 , 3 \% )}$ \\
\hline Hypoglycémie $\mathbf{n}=(\mathbf{\%})$ & $\mathbf{1 0}(\mathbf{9 , 4 \% )}$ \\
\hline Psychogène $\mathrm{n}=(\%)$ & $9(8,5 \%)$ \\
\hline Cardiopathie $\mathrm{n}=(\%)$ & $6(5,7 \%)$ \\
\hline Hypotension orthostatique $\mathrm{n}=(\%)$ & $5(4,7 \%)$ \\
\hline Indéterminée $\mathrm{n}=(\%)$ & $1(0,9 \%)$ \\
\hline
\end{tabular}

En gras, les 3 causes les plus fréquentes. 
Tableau 6. Causes des syncopes définies selon la classification classique [1].

\begin{tabular}{|c|c|}
\hline & Syncopes $n=48(100 \%)$ \\
\hline Origine réflexe dont vagale $n=(\%)$ & $28(58,3 \%)$ \\
\hline Indéterminée $n=(\%)$ & $8(16,7 \%)$ \\
\hline Cardiopathie $n=(\%)$ & $7(14,6 \%)$ \\
\hline Hypotension orthostatique $\mathrm{n}=(\%)$ & $2(4,2 \%)$ \\
\hline Hypoglycémie $\mathrm{n}=(\%)$ & $2(4,1 \%)$ \\
\hline Psychogène $n=(\%)$ & $1(2,1 \%)$ \\
\hline Epilepsie ou crise convulsive $n=(\%)$ & $0(0,0 \%)$ \\
\hline
\end{tabular}

En gras, les 3 causes les plus fréquentes. 
Tableau 7. Causes des syncopes définies selon la nouvelle classification diffusée par la Haute autorité de Santé [5].

\begin{tabular}{|l|c|}
\hline & Syncopes/HAS $\mathrm{n}=97(100 \%)$ \\
\hline Origine réflexe dont vagale $\mathbf{n}=(\mathbf{\%})$ & $\mathbf{6 8}(\mathbf{7 0 , 1 \%})$ \\
\hline Cardiopathie $\mathbf{n}=(\%)$ & $\mathbf{1 2}(\mathbf{1 2 , 4 \% )}$ \\
\hline Indéterminée $\mathbf{n}=(\%)$ & $\mathbf{1 0}(\mathbf{1 0 , 3 \% )}$ \\
\hline Hypotension orthostatique $\mathrm{n}=(\%)$ & $7(7,2 \%)$ \\
\hline
\end{tabular}

En gras, les 3 causes les plus fréquentes. 
Tableau 8. Variables associées à l'hospitalisation parmi les 296 patients de notre étude.

\begin{tabular}{|c|c|c|c|c|}
\hline & $\begin{array}{l}\text { Patients hospitalisés } \\
\qquad \mathrm{n}=67\end{array}$ & $\begin{array}{c}\text { Patients non } \\
\text { hospitalisés } \\
\text { n=229 }\end{array}$ & DM & $p$ \\
\hline $\begin{array}{l}\text { Age (années) } \\
\text { moyenne +/-écart type }\end{array}$ & $59,8+/-19,7$ & $41,0+/-21,0$ & 0 & $<0,0001$ \\
\hline Age $\geq 60$ ans $n=(\%)$ & $39(58,2 \%)$ & $43(18,8 \%)$ & 0 & $<0,0001$ \\
\hline Sexe masculin $n=(\%)$ & $34(50,7 \%)$ & $76(33,2 \%)$ & 0 & 0,008 \\
\hline $\begin{array}{l}\text { Antécédent de cardiopathie } \\
\mathrm{n}=(\%)\end{array}$ & $20(29,9 \%)$ & $27(11,8 \%)$ & 0 & 0,0004 \\
\hline $\begin{array}{l}\text { Antécédent d'épilepsie } n= \\
(\%)\end{array}$ & $9(13,4 \%)$ & $12(5,2 \%)$ & 0 & $0,03 *$ \\
\hline $\begin{array}{l}\text { Traitement hypoglycémiant } \\
\mathrm{n}=(\%)\end{array}$ & $15(22,4 \%)$ & $22(9,6 \%)$ & 0 & 0,005 \\
\hline $\begin{array}{l}\text { Traitement } \\
\text { antihypertenseurs } n=(\%)\end{array}$ & $33(49,3 \%)$ & $48(21,0 \%)$ & 0 & 0,0001 \\
\hline $\begin{array}{l}\text { Traitement psychotrope } \mathrm{n}= \\
(\%)\end{array}$ & $19(28,4 \%)$ & $37(16,2 \%)$ & 0 & 0,02 \\
\hline $\begin{array}{l}\text { Circonstances favorisantes } \\
\mathrm{n}=(\%)\end{array}$ & $15(22,4 \%)$ & $145(63,3 \%)$ & 12 & 0,001 \\
\hline Prodromes $n=(\%)$ & $42(62,7 \%)$ & $183(80,0 \%)$ & 0 & 0,0003 \\
\hline $\begin{array}{l}\text { Perte de connaissance } \\
\text { complète } n=(\%)\end{array}$ & $51(76,1 \%)$ & $103(45,0 \%)$ & 0 & 0,0001 \\
\hline Présence bradycardie $\mathrm{n}=(\%)$ & $10(15,0 \%)$ & $26(11,4 \%)$ & 17 & 0,53 \\
\hline $\begin{array}{l}\text { Examen clinique normal } \mathrm{n}= \\
(\%)\end{array}$ & $23(34,3 \%)$ & $146(63,8 \%)$ & 0 & 0,0001 \\
\hline
\end{tabular}

$\mathrm{DM}=$ données manquantes

*Utilisation du test exact de Fisher 
Tableau 9. Facteurs indépendamment associés à l'hospitalisation en analyse multivariée.

\begin{tabular}{|c|c|c|c|c|}
\hline Variables & P univarié & OR & IC 95\% & P multivarié \\
\hline Age & $<0,0001$ & 1,036 & {$[1,021-1,052]$} & $<0,0001$ \\
\hline $\begin{array}{c}\text { Circonstances } \\
\text { favorisantes }\end{array}$ & 0,0001 & 0,247 & {$[0,120-0,511]$} & 0,0002 \\
\hline $\begin{array}{c}\text { Perte de connaissance } \\
\text { complète }\end{array}$ & 0,0001 & 3,031 & {$[1,604-6,530]$} & 0,002 \\
\hline $\begin{array}{c}\text { Examen clinique } \\
\text { normal }\end{array}$ & 0,0001 & 0,53 & {$[0,274-1,055]$} & 0,07 \\
\hline
\end{tabular}

OR odds ratio, IC 95\% intervalle confiance $95 \%$

Le test d'Hosmer et Lemeshow donne $\mathrm{p}=0,57$ et la c-statistique est à 0,835. 


\section{Légendes des figures}

Figure 1 : Etiologies des malaises.

Figure 2: Diagramme de flux des patients dans l'étude.

${ }^{1}$ SCAM : Sortie contre avis médical 